1. MBBS, FCPS (Urology) Senior Registrar Urology \& Renal Transplantation, Allied Hospital, Faisalabad.

2. MBBS, FCPS (Urology)

Senior Registrar Urology DHQ Hospital, Faisalabad

3. MBBS, MCPS, FCPS, FRCS (Urology) Assistant Professor Urology Allied / DHQ Hospital, Faisalabad Medical University, Faisalabad.

4. MBBS, FCPS (Gen. Surgery), FCPS (Urology)

Associate Professor Urology Allied / DHQ Hospital,

Faisalabad Medical University, Faisalabad.

5. MBBS, FCPS (Urology)

Associate Professor Urology Allied / DHQ Hospital,

Faisalabad Medical University, Faisalabad

6. MBBS, MS (Urology)

Professor Urology Allied / DHQ Hospital,

Faisalabad Medical University, Faisalabad.

7. MBBS, MS (Urology)

Professor Urology

Independent University Hospital, Faisalabad.

Correspondence Address:

Dr. Muhammad Saifullah

Department of Urology,

DHQ Hospital, Faisalabad.

drsaif1987@gmail.com

Article received on:

29/05/2020

Accepted for publication:

$15 / 07 / 2020$

\section{Correlation between bladder wall thickness and IPSS in patients having benign prostatic hyperplasia.}

Moin Anwar ${ }^{1}$, Muhammad Saifullah², Muhammad Tahir Bashir Malik ${ }^{3}$, Muhammad Irfan Munir ${ }^{4}$, Muhammad Akmal $^{5}$, Ghulam Mehboob Subhani ${ }^{6}$, Safdar Hassan Javed

ABSTRACT... Objectives: Our objective was determination of the correlation between mean bladder wall thickness and mean IPSS in BPH diagnosed patients. Study Design: Cross sectional study. Setting: Department of Urology \& Renal Transplantation, Punjab Medical College / Allied Hospital, Faisalabad. Period: Six months from 01-04-2016 to 30-09-2016. Material \& Methods: Total 70 patients were enrolled for study from outpatient department of Urology, AHF. IPSS was calculated and recorded. Transabdominal ultrasound KUB was done to measure bladder wall thickness. Urine complete examination and culture was done to rule out UTI. Existence of bladder tumor was ruled out by previous history and ultrasonography. The data was collected on a Proforma by myself. Results: Out of 70 enrolled cases, $47.14 \%$ $(n=33)$ were aged $50-60$ years whereas $52.86 \%(n=37)$ were $61-70$ years of age. Mean age was calculated as $61.27+5.31$ years. Mean Bladder Wall thickness was $3.64+0.72$ and IPSS was calculated to be $12.84+2.79$ (r0.9056). Correlation between mean bladder wall thickness and mean IPSS in patients having benign prostatic hyperplasia was calculated as significant. Conclusion: In patients suffering from $\mathrm{BOO}$ due to $\mathrm{BPH}$, we have found a very strong positive correlation between mean IPSS and mean BWT.

Key words: $\quad$ Benign Prostatic Hyperplasia, Bladder Wall Thickness, IPSS.

Article Citation: Anwar M, Saifullah M, Malik MTB, Munir MI, Akmal M, Subhani GM, Javed $\mathrm{SH}$. Correlation between bladder wall thickness and IPSS in patients having benign prostatic hyperplasia. Professional Med J 2020; 27(12):2553-2557. https://doi.org/10.29309/TPMJ/2020.27.12.5021

\section{INTRODUCTION}

As the name indicates bladder outlet obstruction (BOO) is a problem in which outflow of the urine from urinary bladder is hindered due to any cause. However, benign prostatic hyperplasia $(\mathrm{BPH})$ is leading cause of BOO especially in ageing males. ${ }^{1,2} 50 \%$ of men above the age of 50 develop $\mathrm{BPH}$ and its incidence increases with every passing year. ${ }^{3}$ Studies conducted on the autopsies have revealed that prevalence of $\mathrm{BPH}$ is $8 \%$ during the $4^{\text {th }}$ decade and gradually increases to $80 \%$ in $9^{\text {th }}$ decade of life. ${ }^{4}$ Although $\mathrm{BPH}$ develops under the influence of many factors including dietary and hormonal factors, evidence shows that genetics also play a strong role in its development. ${ }^{5}$ Physical urethral compression by the prostate results in anatomical BOO. Two mechanisms which contribute to BOO include increasing prostate volume (static component) and increase in tone of stromal smooth muscles (dynamic component). ${ }^{4}$ Symptoms caused by $\mathrm{BOO}$ are called lower urinary tract symptoms (LUTS). These are divided into two varieties which are called storage LUTS and voiding LUTS. Storage symptoms include frequent voiding, urgency and nocturia wheras voiding symptoms are straining to void, poor stream, intermittency and sensation of incomplete evacuation. ${ }^{2}$

Several scoring systems have been developed to quantify severity of LUTS. International Prostate Symptom Score (IPSS) has been widely accepted around the world. It is recommended for routine clinical evaluation of LUTS by many 
organizations including EAU, AUA and $\mathrm{WHO}{ }^{6}$ As IPSS is a subjective indicator, therefore objective assessment tools like prostatic volume, post void residual volume (PVRU), uroflowmetry (UFM) and bladder wall thickness (BWT) have been developed to correctly assess the severity of BO0.7 Rosier et al studied that out of 55 cases having mild IPSS, only $51 \%$ patients were obstructed. ${ }^{8}$

Bladder wall is naturally thicker in males than females. Females have BWT of $3.10 \mathrm{~mm}$ compared to $3.27 \mathrm{~mm}$ in males before voiding. Whereas post-voiding, it changes to $5.18 \mathrm{~mm}$ and 5.39 $\mathrm{mm}$ respectively. Thick male bladder wall is due to the fact that male bladder has to pump against greater resistance due to longer urethra. ${ }^{9}$ When subjected to continuous resistance, detrusor muscle undergoes hypertrophic changes. Apart from this fibroelastic hyperplasia and collagen deposition in the bladder wall also contributes to further thickening of bladder wall. ${ }^{10}$ Mirone et al described that bladder wall hypertrophy is the first anatomic change after the patient develops BOO. ${ }^{11}$ At a volume of $338 \pm 82.1 \mathrm{ml}$, mean BWT is calculated to be $2.0 \pm 0.4 \mathrm{~mm} .{ }^{1}$ Tokgöz Ö described correlation between IPSS and BWT as significant $(r=0.377) .{ }^{12}$

As described IPSS is subjective assessment tool which is extremely dependent on patient's $I Q$ level and literacy, therefore it is very difficult to calculate IPSS accurately. BWT on the other hand is an objective assessment tool which can be easily measured using routine ultrasound KUB. In our study, we want to establish a relation between IPSS and BWT. If established, BWT can be quoted as a better indicator of assessment of LUTS than IPSS.

\section{MATERIAL \& METHODS}

This cross sectional study was conducted at Department of Urology and Renal Transplantation, Allied Hospital Faisalabad (AHF) / Punjab Medical College, Faisalabad for six months between 0104-2016 and 30-09-2016. Patient aged between 50 and 70 years having enlarged prostate diagnosed by ultrasound KUB were taken as study subjects. However, patients having UTI, hydronephrosis or bladder malignancy were ruled out of the study.

After approval of the research topic from the Ethical Review Committee of the Hospital, informed consent was taken from all the enrolled patients. IPSS was calculated in the outpatient Department of Urology, AHF. Ultrasound KUB was done to measure BWT. It was reported by consultant radiologist of Department of Radiology, AHF. Urine complete examination and culture was done to rule out urinary tract infection (UTI). Bladder malignancy was ruled out on the basis of detailed history and ultrasonography. Data was recorded on a proforma.

All data was entered and processed in SPSS v- 17. Numerical variables i.e. age, IPSS, BWT was described with mean \pm standard deviation. Pearson correlation was used to find out correlation between mean BWT and mean IPSS score. A P-value $<0.05$ was considered significant.

\section{RESULTS}

In our study, total 70 patients were included. Age distribution is shown in Figure-1. Mean age \pm sd was calculated as $61.27+5.31$ years.

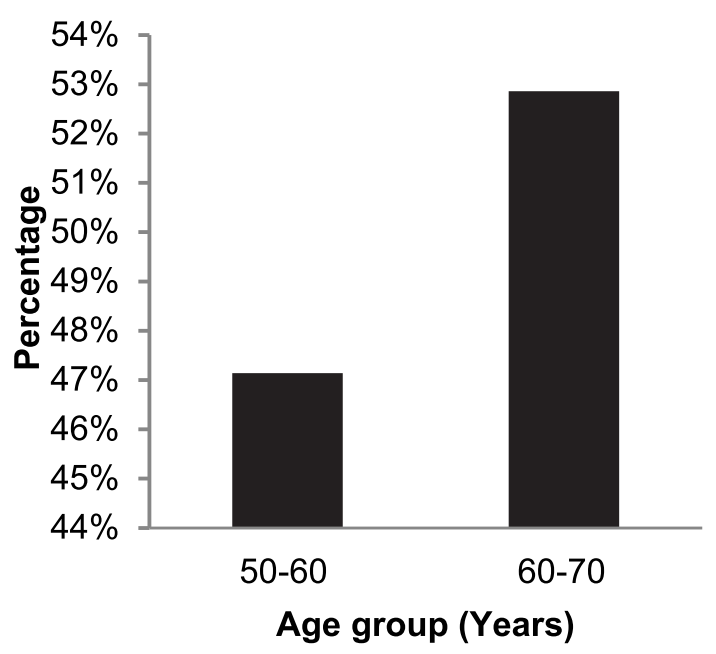

Figure-1. Distribution of patients accord to age

In our study, mean bladder wall thickness was calculated to be $3.64+0.72$ while IPSS was 12.84+2.79. It shows a significant positive correlation. (Table-l) 


\begin{tabular}{|c|c|c|c|}
\hline \multicolumn{2}{|c|}{ Bladder Wall thickness } & \multicolumn{3}{|c|}{ IPSS } \\
\hline Mean & SD & Mean & SD \\
\hline 3.64 & 0.72 & 12.84 & 2.79 \\
\hline
\end{tabular}

Table-I. Correlation between mean IPSS and mean BWT in BPH patients $(n=70)$ $r=0.9056$ $p$ value $=0.00$

\section{DISCUSSION}

Chronic bladder outlet obstruction is a result of many urological problems but BPH leads the rest when it comes to prevalence. Chronically obstructed bladders undergo significant detrusor hypertrophy leading to a densely trabeculated and thickened bladder wall. Patients having predominantly voiding LUTS are often diagnosed having thick walled bladder radiologically. Many urologists have shown keen interest in finding the clinical importance of measuring BWT. Although there is no proven significance of BWT but research is still going on to find its clinical implications. ${ }^{13}$ Transabdominal ultrasound KUB is commonly used investigation which can accurately measure BWT. Low frequency probes are routinely used for this purpose. ${ }^{14}$ However, reporting of BWT has not yet been standardized which is a huge concern for its further utilization as a diagnostic as well as prognostic tool.

We planned this study with the view that IPSS is a subjective measurement of the severity of LUTS. It is a difficult task to quantify the severity of symptoms as it is extremely dependent on the literacy and IQ level of each patient. Whereas BWT is an objective assessment tool which can be used to assess the severity of LUTS using simple, noninvasive and cheap method of transabdominal ultrasound scan. We hypothesized that if BWT increases with an increase in IPSS then it is will establish a correlation between IPSS and BWT. Moreover, BWT may be used to counter check the symptom severity which will be assessed using IPSS.

In our study, mean BWT was calculated as 3.64 \pm 0.72 while IPSS was calculated as $12.84 \pm$ 2.79. Correlation between mean IPSS and mean BWT in patients having BPH was calculated as significant. Mangera $A$ et al calculated mean IPSS of the patients $25.04 \pm 3.34$ whereas mean BWT was $5.07 \pm 0.91 \mathrm{~mm}$. He interpreted that BWT is a good predictor of $\mathrm{BOO}$ instead of IPSS. ${ }^{15}$ YIImaz A et al found a positive correlation between BWT and IPSS. However, he declared it as insignificant and noted that BWT is a better predictor of the duration of obstruction. ${ }^{16}$ Reddy $S$ et al studied BWT in relation to pressure flow studies (PFS) and concluded that it is a promising substitute for PFSs to establish the diagnosis of BOO. It is a relatively quick, cheap, easy to perform and noninvasive method. ${ }^{14}$

Although our results are in agreement with different international studies, still some studies have quoted a poor correlation between IPSS and obstructing prostates. ${ }^{8}$ These studies confer that IPSS is not a good assessment tool for quantifying LUTS.

Franco $G$ et al concluded that concomitant measurement of both BWT and intravesical prostatic protrusion (IPP) has $87 \%$ diagnostic accuracy where detection of $\mathrm{BOO}$ is required. ${ }^{17}$ Furthermore, Manieri et al also reported that increased BWT along with IPP Grade accurately predicts $\mathrm{BOO}$. He conferred that cut off value of $5 \mathrm{~mm}$ BWT can be used to establish a diagnosis of BOO. ${ }^{18}$ Positive correlation of BWT with other indicators of $\mathrm{BOO}$ like post void residual urine (PVRU) and uroflowmetry has also been successfully studied by Panay DC et al. ${ }^{19}$

BWT also includes mucosal thickness which is usually increased in response to inflammations as well as malignancies, whereas BOO affects detrusor muscle predominantly. Exclusion of mucosal thickness from BWT gives us detrusor wall thickness (DWT). BWT measurement gives an indirect idea of the DWT. However direct DWT measurement has used as a better alternative to BWT. However it requires expertise as well as experience. $100 \%$ sensitivity has been achieved by using a higher DWT cut-off i.e., more than $2.9 \mathrm{~mm}$. It helps in avoidance of more invasive investigations like urodynamic studies. ${ }^{20}$

Tokgöz Ö et al significantly correlated IPSS with DWT. According to his study, individual parameters 
like DWT and PVRU values showed positive and strong correlations with IPSS (Spearman's correlation coefficients were 0.463 and 0.623 , respectively). ${ }^{12}$ Okele et al also concluded DWT assessment is a better investigation to detect $\mathrm{BOO}$ in comparison to PVRU, prostate size and urodynamic studies. $89 \%$ results of DWT and PFS were in agreement according to him. 94\% positive predictive value (PPV) was achieved using DWT $\geq 2 \mathrm{~mm} \cdot{ }^{21}$ Kessler et al proved that patients having obstructing prostates had significantly higher DWT than patients without BOO. He observed that DWT > $2.9 \mathrm{~mm}$ had a 100\% PPV in diagnosing $\mathrm{BOO}{ }^{22}$

Although many authors have claimed BWT and DWT are very helpful in diagnosing BOO but there are few limitations. Some authors rightfully claim that BWT is dependent on the degree of bladder filling which leads to difference in calculations even of the same patient at different times ${ }^{23}$. For this reason, BWT as well as DWT require standardization. Certain standardization methods have been proposed by Incontinence Research Society which include proper information regarding bladder filling volume, ultrasound probe frequency and area of bladder measured (BWT vs. DWT). Provision of an ultrasound picture with clear marker positioning has also been advised. In clinical practice, BWT and DWT can be declared useful to quantify detrusor hypertrophy due to BOO only after adopting standardized techniques.

\section{CONCLUSION}

In patients suffering from $\mathrm{BOO}$ due to $\mathrm{BPH}$, we have found a very strong positive correlation between mean IPSS and mean BWT. However, bigger studies using standardized techniques are required to establish this fact.

Copyright@ 15 July, 2020.

\section{REFERENCES}

1. Kanyilmaz S, Calis FA, Cinar Y, Akkoc Y. Bladder wall thickness and ultrasound estimated bladder weight in healthy adults with portative ultrasound device. J Res Med Sci. 2013; 18:103-6.
2. Sharis OS, Zulkifli MZ, Hamzaini AH. Predicting outcome of trial of voiding without catheter in acute urinary retention with intravesical prostatic protrusion. Malays J Med Sci. 2013; 20:56 $\square$ 9.

3. Kim EH, Larson JA, Andriole GL. Management of benign prostatic hyperplasia. Ann Rev Med. 2016; $67: 1,137-51$.

4. Patel ND, Parsons JK. Epidemiology and etiology of benign prostatic hyperplasia and bladder outlet obstruction. Indian J Urol. 2014; 30:170-6.

5. Lim KB. Epidemiology of clinical benign prostatic hyperplasia. Asian J Urol. 2017; 4:148-51.

6. Kim JH, Shim JS, Choi H, et al. Terminal dribbling in male patients with lower urinary tract symptoms: Relationship with international prostate symptom score and with intravesical prostatic protrusion. BMC Urol. 2015; 15:89.

7. De Nunzio C, Autorino R, Bachmann A, Briganti A, Carter $S$, Chun $F$. The diagnosis of benign prostatic obstruction: Development of a clinical nomogram. Neurourol Urodyn. 2014 Dec 18.

8. Foo KT. Solving the benign prostatic hyperplasia puzzle. Asian J Urol. 2016; 3:6-9.

9. Idigo FU, Agu Cl, Okeji MC, Anakwue AC, Nwogu UB. Sonographic determination of urinary bladder wall thickness in a Healthy Nigerian Population. World $\mathrm{J}$ Med Sci. 2017; 14:33-8.

10. Karakose A, Aydogdu O, Atesci YZ. The relationship between bladder wall thickness and lower urinarytract symptoms: Does bladder wall thickness change after alpha blocker therapy with alfuzosin? Can Urol Assoc J. 2014; 8:26-9.

11. Mirone V, Imbimbo C, Longo N, Fusco F. The detrusor muscle: An innocent victim of bladder outlet obstruction. Eur Urol. 2007; 51:57-66.

12. Tokgöz Ö, Tokgöz H, Ünal í, Delibaş U, Yıldız S, Voyvoda N, Erdem Z. Diagnostic values of detrusor wall thickness, postvoid residual urine, and prostate volume to evaluate lower urinary tract symptoms in men. Diagn Interv Radiol. 2012; 18:277-81.

13. Mangat R, Ho HSS, Kuo TLC. Non-invasive evaluation of lower urinary tract symptoms (LUTS) in men. Asian J Urol. 2018; 5:42-7.

14. Reddy S, Shaik A. Non-invasive evaluation of bladder outlet obstruction in benign prostatic hyperplasia: A clinical correlation study. Arab J Urol. 2019; 17:25964. 
15. Mangera A, Osman NI, Chapple CR. Assessment of BPH/BOO. Indian J Urol. 2014; 30:177-80.

16. Yılmaz A, Aslan $Y$, Uzun B, Tekdoğan U, Tuncel A, Atan A. Relationship between bladder wall thickness and duration of symptoms, uroflowmetry parameters, and international prostate symptom score in patients with lower urinary tract symptoms. Turk J Urol. 2009; 35: 361-5.

17. Franco G, De Nunzio C, Leonardo C, et al. Ultrasound assessment of intravesical prostatic protrusion and detrusor wall thickness-new standards for noninvasive bladder outlet obstruction diagnosis? J Urol. 2010; 183:2270-4.

18. Manieri C, Carter SS, Romano G, et al. The diagnosis of bladder outlet obstruction in men by ultrasound measurement of bladder wall thickness. J Urol. 1998; 159:761-5.

19. Panayi DC, Khullar V, Fernando R, Tekkis P. Transvaginal ultrasound measurement of bladder wall thickness: A more reliable approach than transperineal and transabdominal approaches. BJU Int. 2010; 106:151922.
20. Lee $\mathrm{H}$, Choo M, Kim M, et al. Changes in bladder wall thickness and detrusor wall thickness after surgical treatment of benign prostatic enlargement in patients with lower urinary tract symptoms: A preliminary report. Korean J Urol. 2014; 55:47-51.

21. Oelke M, Höfner K, Jonas U, de la Rosette J, Ubbink $D$, Wijkstra $H$. Diagnostic accuracy of noninvasive tests to evaluate bladder outlet obstruction in men: Detrusor wall thickness, uroflowmetry, postvoid residual urine, and prostate volume. European Urology. 2007; 52:827-35.

22. Kessler TM, Gerber R, Burkhard FC, Studer UE, Danuser $\mathrm{H}$. Ultrasound assessment of detrusor thickness in men-can it predict bladder outlet obstruction and replace pressure flow study? J Urol. 2006; 175:21703.

23. Brkljacić B, Kuzmić AC, Dmitrović R. Ultrasoundestimated bladder weight in healthy children. Eur Radiol. 2004; 14:1596-9.

\begin{tabular}{|c|c|c|c|}
\hline \multicolumn{4}{|c|}{ AUTHORSHIP AND CONTRIBUTION DECLARATION } \\
\hline Sr. \# & Author(s) Full Name & Contribution to the paper & Author(s) Signature \\
\hline 2 & $\begin{array}{l}\text { Moin Anwar } \\
\text { Muhammad Saifullah }\end{array}$ & $\begin{array}{l}\text { Principal author, Data collectior } \\
\text { and Manuscript writer. } \\
\text { Investigator, Data Collector. }\end{array}$ & wat \\
\hline 3 & M. Tahir Bashir Malik & Literature review. & 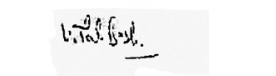 \\
\hline 4 & M. Irfan Munir & Statistical analysis. & \\
\hline 5 & Muhammad Akmal & Resutls compilation. & \\
\hline 6 & $\begin{array}{l}\text { Ghulam Mehboob } \\
\text { Subhani }\end{array}$ & Final layout. & \\
\hline 7 & Safdar Hassan Javed & $\begin{array}{l}\text { Proof reading \& Research } \\
\text { supervisor. }\end{array}$ & $\delta+1 \leq$ \\
\hline
\end{tabular}

